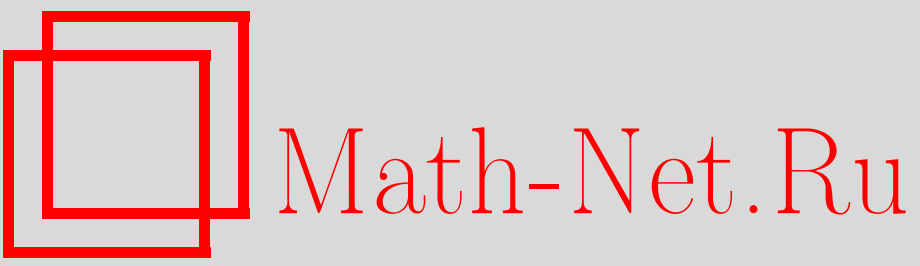

А. О. Пришляк, Сопряженность функций Морса на 4-мерных многообразиях, УМH, 2001, том 56, выпуск 1, 173-174

DOI: https://doi.org/10.4213/rm370

Использование Общероссийского математического портала Math-Net.Ru подразумевает, что вы прочитали и согласны с пользовательским соглашением

http://www.mathnet.ru/rus/agreement

Параметры загрузки:

IP: 52.6 .47 .48

26 апреля 2023 г., 06:25:55 


\section{СОПРЯЖЕННОСТЬ ФУНКЦИЙ МОРСА НА 4-МЕРНЫХ МНОГООБРАЗИЯХ}

\section{А. О. Пришляк}

Пусть $M$ - гладкое многообразие, $f$ и $g$ - функции Морса на нем. Функции $f$ и $g$ назьваются сопряженными, если существуют гомеоморфизмы $h: M \rightarrow M, h^{\prime}: \mathbb{R}^{1} \rightarrow \mathbb{R}^{1}$ такие, что $f h^{\prime}=g h$ и гомеоморфизм $h^{\prime}$ сохраняет ориентацию.

В работах [1], [2] получены критерии сопряженности функций Морса на двумерных, а в [3] на трехмерных многообразиях. Если сопрягающие гомеоморфизмы суть изотопные тождественному диффеоморфизмы, то в случае односвязных многообразий размерности болшше 5 в [4] дан критерий сопряженности функций Морса в терминах эквивалентности упорядоченных базисных цепных комплексов.

1. Разложения на ручки с воротниками. Разложением на ручки с воротниками называется последовательность вложений $M_{0} \subset M_{0}^{\prime} \subset M_{1} \subset M_{1}^{\prime} \subset M_{2} \subset \cdots \subset M_{N}=M$ таких, что $M_{0}$ есть объединение $n$-мерных дисков (0-ручек), $M_{i}^{\prime}$ получается из $M_{i}$ с помощью приклейки воротника $N_{i} \times[0,1]$, где $N_{i}=\partial M_{i}$, а $M_{i+1}$ получается из $M_{i}^{\prime}$ с помощью приклейки ручек. На каждом воротнике задана проекция $\pi: N_{i} \times[0,1] \rightarrow[0,1]$.

Разложения на ручки с воротниками назьваются изоморфными, если существует гомеоморфизм между многообразиями, который переводит ручки в ручки, воротники в воротники, сохраняя разбиение воротников на слои.

По каждой функции Морса $f: M \rightarrow \mathbb{R}^{1}$ с критическими значениями $\{1,2, \ldots, N\}$ зададим разложение на ручки с воротниками так, что при этом внутренности воротников $N_{i} \times[0,1]$ будут послойно гомеоморфны компонентам связности множества $M \backslash f^{1}(\{1,2, \ldots, N\})$. Функции будут сопряженными тогда и только тогда, когда соответствующие разложения на ручки с воротниками изоморфны [3].

2. Критерий сопряженности функций Морса. Зададим на каждом воротнике такую структуру прямого произведения $N_{i} \times[0,1]$, что для каждого $t \in(0,1) N_{i} \times\{t\}=f^{-1}(y)$ для соответствующего регулярного значения $y$. Тогда после удаления из многообразия воротников и отождествления $N_{i} \times\{0\}$ с $N_{i} \times\{1\}$ получим разложение на ручки без воротников. Это разложение зависит от того, как мы зададим структуру прямого произведения на каждом воротнике. При этом разным структурам прямого произведения соответствуют приклейки ручек по изотопным вложениям.

$\mathrm{C}$ помощью изотопий средних сфер любое разложение на ручки (без воротников) можно привести к такому, у которого средние и косредние сферы ручек пересекаются трансверсально, и такому, что каждая ручка приклеивается к объединению ручек меньшей размерности. Такие разложения на ручки будем назьвать простыми.

Разложение на ручки называется упорядоченным, если задано отображение множества ручек на множество $\{1,2, \ldots, N\}$. При этом соответствующее значение назовем номером ручки. Для разложения на ручки, построенного по функции Морса, ее номер есть порядковый номер (по возрастанию) соответствующего критического значения.

Упорядоченные простые разложения на ручки (УПРР) назьваются изоморфньмии, если существует гомеоморфизм многообразий, который переводит ручки в ручки, средние и косредние диски соответственно, сохраняя при этом упорядочение ручек. Обозначим $M^{k}$ объединение ручек, индекс которых не превосходит $k$ и $L^{k}=\partial M^{k}$. Из построения и результатов работы [3] следует

Теорема 1. Две функции Морса будут сопряженными тогда и только тогда, когда из УПРР первой функции можно получить УПРР, изоморфное УПРР второй функции, при помощи

1) изотопий в $L^{k}$ вложений средних сфер с носителем в границе обвединения ручек с меньшими номерами; 
2) замены ручки $H_{i}$ на сумму $H_{i} \sharp H_{j}$, если номер $H_{i}$ больше $H_{j}$ (здесь ручки имеют одинаковыи индекс), при этом новая ручка $H_{i} \sharp H_{j}$ имеет тот же номер, что $u H_{i}$.

3. Функции Морса на 4-мерных многообразиях и диаграммы Кирби. Будем рассматривать функции Морса на 4-мерных многообразиях, у которых один минимум и один максимум и нет критических точек индекса 3 . Для каждой такой функции построим упорядоченное простое разложение на ручки. Диаграммы Кирби это такое зацепление в $S^{3}$, в котором каждая вложенная окружность обладает оснашением (задается целым числом) либо является незаузленной окружностью с точкой. Тогда УПРР будет задавать диаграмму Кирби, в которой каждой вложенной окружности с точкой соответствует 1-ручка, а окружности с оснащением - 2-ручка (эта окружность является средней сферой 2-ручки) [5]. При этом каждая окружность будет иметь тот же номер, что и соответствующая критическая точка функции Морса (равньй номеру соответствующей ручки).

Диаграмму Кирби, в которой каждой окружности приписан номер, назовем упорядоченной. Две упорядоченные диаграммы Кирби назьваются изоморфньми, если существует гомеоморфизм сфер $S^{3}$, который отображает вложенные окружности на окружности, сохраняя точки или оснащения, а также их номера.

Упорядоченные диаграммы Кирби назовем эквивалентными, если из одной диаграммы можно получить диаграмму, изоморфную второй, последовательностью таких операций:

1) заменой вложенной оснащенной окружности $u_{i}$ на сумму окружностей $u_{i} \sharp u_{j}$; если при этом $u_{j}$ - также оснащенная окружность, то ее номер должен быть меньше номера $u_{i}$;

2 ) заменой вложенной окружности с точкой $u_{i}$ на сумму окружностей $u_{i} \sharp u_{j}$ при условии, что $u_{j}$ - также окружность с точкой и ее номер меньше номера $u_{i}$.

Из теоремы 1 и определения эквивалентности упорядоченных диаграмм Кирби следует

Теорема 2. Две функции Морса, у которых по одному минимуму и максимуму и нет критических точек индекса 3 , на четырехмерных многообразиях сопряжень тогда и только тогда, когда построенные по ним упорядоченные диаграммы Кирби эквивалентнь.

ЗАмечАниЕ. Теорема будет верна, если все гомеоморфизмы будут гладкими, т.е. диффеоморфизмами.

\section{СПИСОК ЛИТЕРАТУРЫ}

[1] V. V. Sharko // On topological equivalence Morse functions on sufraces. Internat conference at Chelyabinsk State Univ.: Low-Dimensional Topology and Combinatorial Group Theory, 1996. P. 19-23. [2] E. V. Kulinich // Methods Funct. Anal. Topology. 1998. V. 4. № 1. P. 59-64. [3] А.О. Пришляк. Сопряженность функций Морса // Совр. вопросы матем. (Ин-т математики АН Украины, Киев). 1998. С. 94-103. [4] В. В. Шарко. Функции на многообразиях (алгебраические и топологические аспекты). Киев: Наукова думка, 1998. [5] R. C. Kirby. The Topology of 4-Manifolds. Berlin: Springer-Verlag, 1989. 